\title{
Internal transcribed spacer sequence-based rapid molecular identification of Prototheca zopfii and Prototheca blaschkeae directly from milk of infected cows
}

\author{
S. Marques, ${ }^{*} \dagger^{1}$ V. A. R. Huss, $\ddagger$ K. Pfisterer, $\ddagger$ C. Grosse,$\ddagger$ and G. Thompson ${ }^{*} \dagger$ \\ *Centro de Investigação em Biodiversidade e Recursos Genéticos Universidade do Porto (CIBIO/UP), InBio Laboratório Associado Campus \\ Agrário de Vairão, Rua Padre Armando Quintas, n 7, 4485-661 Vairão, Portugal \\ †Laboratório de Doenças Infecciosas do Departamento de Clínicas Veterinárias, Instituto de Ciências Biomédicas de Abel Salazar (ICBAS), \\ Universidade do Porto, Rua de Jorge Viterbo Ferreira, n 228, 4050-313 Porto, Portugal \\ ‡Department of Biology, Molecular Plant Physiology, University of Erlangen-Nürnberg, Staudtstrasse 5, D-91058 Erlangen, Germany
}

\section{ABSTRACT}

The increasing incidence of rare mastitis-causing pathogens has urged the implementation of fast and efficient diagnostic and control measures. Prototheca algae are known to be associated with diseases in humans and animals. In the latter, the most prevalent form of protothecosis is bovine mastitis with Prototheca zopfii and Prototheca blaschkeae representing the most common pathogenic species. These nonphotosynthetic and colorless green algae are ubiquitous in different environments and are widely resistant against harmful conditions and antimicrobials. Hence, the association of Prototheca with bovine mastitis represents a herd problem, requiring fast and easy identification of the infectious agent. The purpose of this study was to develop a reliable and rapid method, based on the internal transcribed spacer (ITS) sequences of ribosomal DNA, for molecular identification and discrimination between P. zopfii and P. blaschkeae in bovine mastitic milk. The complete ITS sequences of 32 Prototheca isolates showed substantial interspecies but moderate intraspecies variability facilitating the design of species-specific PCR amplification primers. The species-specific PCR was successfully applied to the identification of $P$. zopfii and P. blaschkeae directly from milk samples. The intraspecific ITS phylogeny was compared for each species with the geographical distribution of the respective Prototheca isolates, but no significant correlation was found.

Key words: Prototheca spp. bovine mastitis, internal transcribed spacer (ITS), rapid molecular identification, species-specific PCR (ssPCR), geographical distribution

Received December 21, 2014.

Accepted January 23, 2015.

${ }^{1}$ Corresponding author: saramarques@mail.icav.up.pt

\section{INTRODUCTION}

Members of the genus Prototheca are ubiquitous nonphotosynthetic green algae that are associated with pathologies in humans and animals (DiPersio, 2001; Möller et al., 2007; Marques et al., 2008). Presently, 6 species are assigned to the genus Prototheca, with Prototheca zopfii, Prototheca wickerhamii, Prototheca blaschkeae, and Prototheca cutis being identified as pathogenic (Jánosi et al., 2001; Roesler et al., 2006; Satoh et al., 2010). Prototheca zopfii and P. blaschkeae have been mostly associated with clinical or subclinical cases of bovine mastitis (Möller et al., 2007; Marques et al., 2008; Jagielski et al., 2011). Prototheca zopfii was classified into 2 genotypes, genotype 1 and 2, after biochemical, serological, and genetic analyses. Genotype 2 was assigned to be the most prevalent responsible for bovine protothecal mastitis, whereas genotype 1 was considered to be not pathogenic (Roesler et al., 2006; Möller et al., 2007). Prototheca blaschkeae was primarily isolated from a human onychomycosis (Roesler et al., 2006), and then for the first time from bovine mastitis in Portugal (Marques et al., 2008, 2010; Thompson et al., 2009); meanwhile, P. blaschkeae incidences have been reported globally (Jagielski et al., 2010; Gao et al., 2012; Sobukawa et al., 2012; Ricchi et al., 2013).

Prototheca bovine mastitis is increasing worldwide and is currently recognized as endemic. Moreover, this pathology can lead to significant economic losses for the dairy herds and poses a public health problem (Jánosi et al., 2001; Malinowski et al., 2002; Onozaki et al., 2013). No therapy exists to eradicate this infection, and drying off the affected quarter and isolation or culling the infected animals are the only effective counteractions presently applied (Roesler and Hensel, 2003).

Identification of Prototheca species is generally performed by phenotypic and genotypic methods. Whereas phenotypic methods often fail in the correct identifi- 
cation, molecular methods such as PCR amplification with subsequent sequencing of $18 \mathrm{~S}$ or $28 \mathrm{~S}$ ribosomal DNA (rDNA) or RFLP analysis can reliably identify Prototheca spp. (Ueno et al., 2003, 2005; Möller et al., 2007; Marques et al., 2008). Moreover, a real-time PCR was developed to identify different genotypes of P. zopfii (Ricchi et al., 2011). Still, these methods are rather time-consuming and some are costly. Recently, Cremonesi et al. (2012) developed an identification method to differentiate $P$. zopfii genotype 2 from other microalgae. Although this method is rapid, easy, and highly reproducible, it has some limitations, such as temperature and $\mathrm{pH}$ sensibility and limitation of the DNA fragment size.

The most convenient way of genotyping Prototheca isolates with minimal effort seems to be a PCR approach with genotype-specific primers. Such an approach was developed for $P$. zopfii biotypes 1 and 2 and P. blaschkeae by Roesler et al. (2006) and Jagielski et al. (2011) based on 18S rDNA sequences. However, the conserved 18S rDNA offers little choice for selection of genotype-specific primers. The primer pairs applied yielded PCR products of 150, 165, and $126 \mathrm{bp}$, respectively, too similar to be reliably distinguished on conventional gels without additional controls. In an attempt to improve molecular identification of Prototheca, recently Capra et al. (2014) were able to amplify chloroplast and mitochondrial target sequences by a multiplex PCR approach.

In contrast to the conserved $18 \mathrm{~S}$ and $28 \mathrm{~S}$ rDNA, the ribosomal internal transcribed spacer (ITS) region is much more variable and therefore useful for differentiation of strains (Coleman and Mai, 1997). The ITS analysis is now widely applied for phylogenetic studies of closely related Chlorella-like algae (Bock et al., 2010; Krienitz et al., 2011). Attempts to consistently amplify the complete ITS region (ITS1, 5.8S rDNA, and ITS2) of Prototheca were unsuccessful in the past in our hands. Only recently were the first Prototheca ITS sequences published by Hirose et al. (2013) for $P$. wickerhamii.

The aim of the current study was to modify PCR conditions for the successful amplification of ITS sequences from $P$. zopfii genotype 2 and $P$. blaschkeae to determine the genotypic variability and study the correlation of geographical distribution versus phylogeny within 32 strains isolated from milk of infected cows in the north of Portugal. The species-specific (ss) differences of the ITS sequences should then be used to develop a rapid and simple molecular diagnostic tool, based on ssPCR primers, to conclusively identify both Prototheca species directly from milk samples.

\section{MATERIALS AND METHODS}

\section{Prototheca Isolates}

The 32 Prototheca isolates used in our study belong to a major collection of several milk pathogens retrieved between 2002 and 2009 from the milk of cows with mastitis originating from different dairy herds from the north of Portugal (Figure 1). Eighteen strains were identified as $P$. zopfii genotype 2 and 14 as $P$. blaschkeae as described in a previous study (Marques et al., 2008). In addition, 20 samples of bovine mastitic milk (11 infected by $P$. zopfii genotype 2 and 9 by $P$. blaschkeae) and 4 samples of noninfected bovine milk from different dairy herds from the north and center of Portugal were used for DNA isolation. The 11 samples containing $P$. zopfii genotype 2 and the noninfected milk samples were collected during 2014; the 9 samples with P. blaschkeae were from 2007 to 2009. Noninfected milk samples were defined as milk samples retrieved from cows without mastitis or any other infection and with less than 100,000 cells/mL (Guidelines of the National Mastitis Council: Smith et al., 2001). All milk samples were spread on 5\% sheep blood agar (bioMérieux, Marcy l'Etoile, France) and incubated for 24 to $72 \mathrm{~h}$ at $37^{\circ} \mathrm{C}$. Colonies resembling Prototheca were transferred and spread on Sabouraud Dextrose agar medium (Merck Laboratories, Darmstadt, Germany), incubated for another 48 to $72 \mathrm{~h}$ at $37^{\circ} \mathrm{C}$, and identified microscopically thereafter.

\section{Genomic DNA Extraction, ITS Amplification, and Sequencing}

Prototheca genomic DNA preparations were essentially performed as described by Cremonesi et al. (2006, 2012), except that the centrifugation speed of all steps was increased to $1,000 \times g$ at $4^{\circ} \mathrm{C}$ (centrifugation for 15 to $20 \mathrm{~min}$ for the lysis step and $5 \mathrm{~min}$ for the elution step); washing time was increased to $1 \mathrm{~min}$. . Briefly, this protocol makes use of lysing the pellet of cultured cells or centrifuged milk samples with a guanidine thiocyanate containing lysis buffer, in which few colonies were suspended in $500 \mu \mathrm{L}$ of lysis buffer or 500 $\mu \mathrm{L}$ of milk; samples were washed twice with sterilized saline solution and then resuspended in lysis buffer. Then, free DNA was adsorbed to silica contained in a binding solution, washed with ethanol/isopropanol, and desorbed by an elution buffer. The DNA content was then measured in a NanoDrop ND-1000 UV-visible Spectrophotometer (ThermoScientific, Waltham, MA).

For amplification of the complete ITS region the conserved eukaryote-specific primer 1400 forward (Elwood 

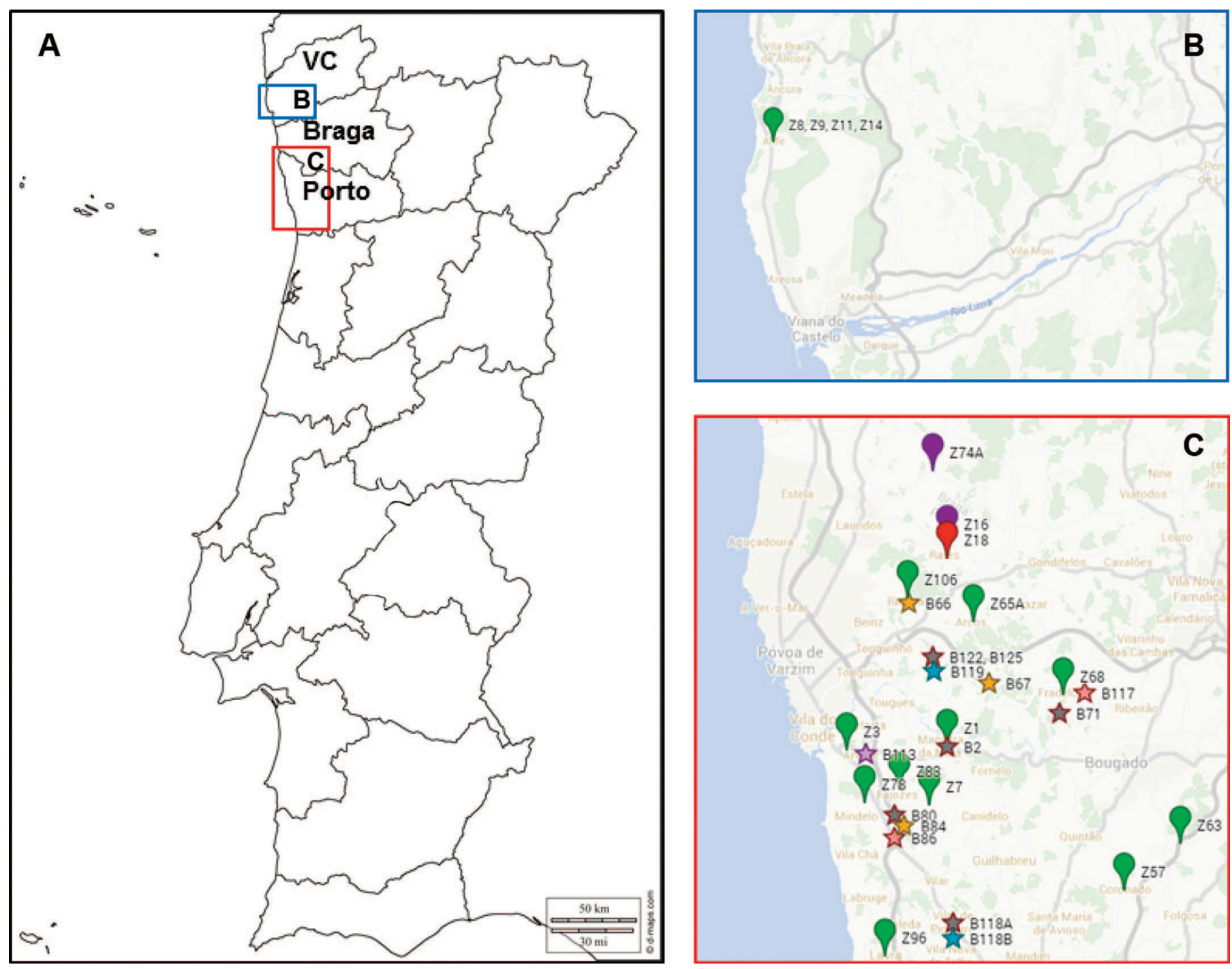

Figure 1. Geographical map of Portugal displaying the origin of Prototheca isolates. (A) Overview and (B and C) detailed maps of the Viana do Castelo (VC), and Braga and Porto districts. Prototheca zopfii (Z) and Prototheca blaschkeae (B) strains are color coded according to the clades determined in the phylogenetic analyses (Figure 2). Different shades of gray were set according to the clades determined in the phylogenetic analyses to discern the isolates within clusters, as shown in Figure 2. Adapted from Google Maps. Color version available online.

et al., 1985), located near the $3^{\prime}$ end of $18 \mathrm{~S}$ rDNA, was modified and renamed as 1400Fm (5'-CCTTTGTACACACCGCCCGTC-3'). This primer was used together with the universal reverse primer NLR 204/21 (5'-ATATGCTTAARTTCAGCGGGT-3; Van der Auwera et al., 1994), located at the $5^{\prime}$ end of $28 \mathrm{~S}$ rDNA. Due to the high GC content of Prototheca (Huss et al., 1988), the PCR conditions were adjusted to allow complete melting of the DNA double strands during the denaturation steps. Thirty nanograms of genomic DNA was amplified in $20 \mu \mathrm{L}$ of reaction volume containing 5 pmol of each primer, $10 \mathrm{~m} M \mathrm{dNTP}, 4 \mu \mathrm{L}$ of $5 \times$ Phusion GC Buffer, 0.2 $\mu \mathrm{L}$ of Phusion High-Fidelity DNA Poly- merase $(2 \mathrm{U} / \mu \mathrm{L})$, and $5 \%$ dimethyl sulfoxide (DMSO; ThermoScientific) in a BioRad C1000 Thermal Cycler (Bio-Rad Laboratories, Hercules, CA). After initial denaturation of $5 \mathrm{~min}$ at $98^{\circ} \mathrm{C}, 35$ cycles were run with $15 \mathrm{~s}$ of denaturation at $98^{\circ} \mathrm{C}, 30 \mathrm{~s}$ of annealing at $57^{\circ} \mathrm{C}$, and $60 \mathrm{~s}$ of extension at $72^{\circ} \mathrm{C}$, followed by another 5 min at $72^{\circ} \mathrm{C}$ after the last cycle. The amplified products were analyzed on a $1.8 \%$ (wt/vol) agarose gel stained with GelRed (Biotium Inc., Hayward, CA) and visualized under UV light (Bio-Rad Laboratories). The PCR products were purified with a QIAquick PCR purification kit (Qiagen, Hilden, Germany) and cloned with a Zero Blunt Topo PCR Cloning Vector Kit (Invitrogen, 
Carlsbad, CA). The insert nucleotide sequences were determined with the universal primers M13 forward and reverse by GATC-Biotech (Constance, Germany).

\section{Phylogenetic Analyses}

The ITS sequences determined in our study were manually aligned on a MicroVAX computer (Digital Equipment Corporation, Maynard, MA) with the sequence editor program distributed by Olsen et al. (1992). For the phylogenetic analyses, the sequences were aligned with sequences of 2 trebouxiophycean green algae, Chlorella vulgaris and Closteriopsis acicularis, which were used as outgroups (Figure 2). To improve the alignment of the ITS data set (ITS1 + 5.8S rDNA + ITS2), secondary structure models were constructed for ITS1 and ITS2 according to the models provided by Coleman and Mai (1997) and Bock et al. (2010). Phylogenetic trees were inferred from all positions of the aligned sequence data by the neighbor-joining (NJ), the maximum parsimony (MP), and the maximum likelihood (ML) method using the PAUP program (Swofford, 2002) for NJ and $\mathrm{MP}$, and randomized axelerated maximum likelihood (RaxML; Stamatakis et al., 2008) for ML. Each 1,000 bootstrap replicates were carried out for NJ, MP, and ML. GenBank Accession Numbers of the ITS sequences determined in this study are listed in Table 1.

\section{SsPCR}

Consensus sequences of the $P$. zopfii genotype 2 and $P$. blaschkeae ITS alignments were checked for PCR primer binding sites, which (1) were specific for each species and (2) at the same time generated PCR products of sufficient size difference to be easily discerned on an agarose gel. From several combinations tested, the following primer pairs for P. zopfii and P. blaschkeae, respectively, proved to be most successful: Pz-ITS-F (5'-TTCGACCGAACGAAACGA- $3^{\prime}$ ) and Pz-ITS-R (5'-AATTCCTGGCATTGGCGACA-3'), as well as PbITS-F (5'-AAGGCCCTGCGTTCTTCGCA-3') and Pb-ITS-R (5'-GCGTGTTCCCGACCGAGAGA-3'). Genomic DNA extracted from Prototheca colonies or directly from infected bovine milk was amplified in 20$\mu \mathrm{L}$ reactions containing $10 \mathrm{pmol}$ of each specific primer pair, $10 \mathrm{~m} M \mathrm{dNTP}, 4 \mu \mathrm{L}$ of $5 \times$ Phusion GC Buffer, and $0.2 \mu \mathrm{L}$ of Phusion High-Fidelity DNA Polymerase $(2 \mathrm{U} / \mu \mathrm{L})$. After initial denaturation for $5 \mathrm{~min}$ at $98^{\circ} \mathrm{C}$, 35 cycles were run with $30 \mathrm{~s}$ of denaturation at $98^{\circ} \mathrm{C}, 30$ $\mathrm{s}$ of annealing at $69^{\circ} \mathrm{C}$, and $30 \mathrm{~s}$ of extension at $72^{\circ} \mathrm{C}$, followed by another $5 \mathrm{~min}$ at $72^{\circ} \mathrm{C}$ after the last cycle. The amplification products were analyzed on a $1.8 \%$ (wt/vol) agarose gel as described previously.

\section{RESULTS}

\section{ITS Amplification and Sequencing, Phylogenetic Analyses, and Correlation Between Subclades and Geographical Distribution of Infected Herds}

The nuclear DNA of Prototheca spp. has an exceptional high molar $\mathrm{G}$ and $\mathrm{C}$ content of about 70 to $75 \%$ (Huss et al., 1988). The corresponding high melting temperature may lead to incomplete melting of the DNA during the PCR denaturation steps, thereby explaining the failure of previous attempts to reliably amplify the ITS region of Prototheca spp. under standard conditions. We modified the PCR protocol mainly by adding DMSO, which is known to lower the melting temperature of DNA (Escara and Hutton, 1980). Using this protocol with the conserved amplification primers 1400Fm and NLR 204/21, which comprise the complete ITS region, we could successfully amplify and sequence this region from $18 P$. zopfii genotype 2 and 14 P. blaschkeae strains isolated from bovine mastitic milk. The complete ITS length varied from 696 to 744 nucleotides (nt) between strains of $P$. zopfii genotype 2 , and from 1,086 to 1,096 nt between P. blaschkeae strains. Thus, the intraspecies variability was more pronounced in $P$. zopfii genotype 2 , although its ITS was up to $400 \mathrm{nt}$ shorter. The extra nucleotides of the $P$. blaschkeae ITS could mainly be allocated to the extension of helix 1 of ITS1 and helix 4 of ITS2 according to the secondary structure models of Coleman and Mai (1997) and Bock et al. (2010). Under consideration of the secondary structure, the ITS sequences could unambiguously be aligned and all positions used for the phylogenetic analyses. The ML tree depicted in Figure 2 clearly discriminates both species with high statistical support. For the graphical representation of the tree, we preferred a cladogram to a phylogram, as the intraspecies variability, especially in P. blaschkeae, is rather low. Nevertheless, within each species some strains cluster together with high or moderate bootstrap support, indicating independent evolution of subclades. It would be interesting to know if the existence of such subclades could be used to trace infection pathways between herds. We therefore tried to correlate, for both species, the occurrence of specific clades obtained by the phylogenetic analyses with the geographical distribution of the herds from which the strains were isolated (Figures 1 and 2). However, no obvious correlation was found. Both Prototheca spp. coexist in the same geographical regions and, except for one case, no tendency for the occurrence of a specific subclade only within a restricted area could be deduced. The 3 strains of 1 P. zopfii genotype 2 subclade (Z16, Z74A, and Z18) were found in 2 herds only $4 \mathrm{~km}$ apart, but the small 


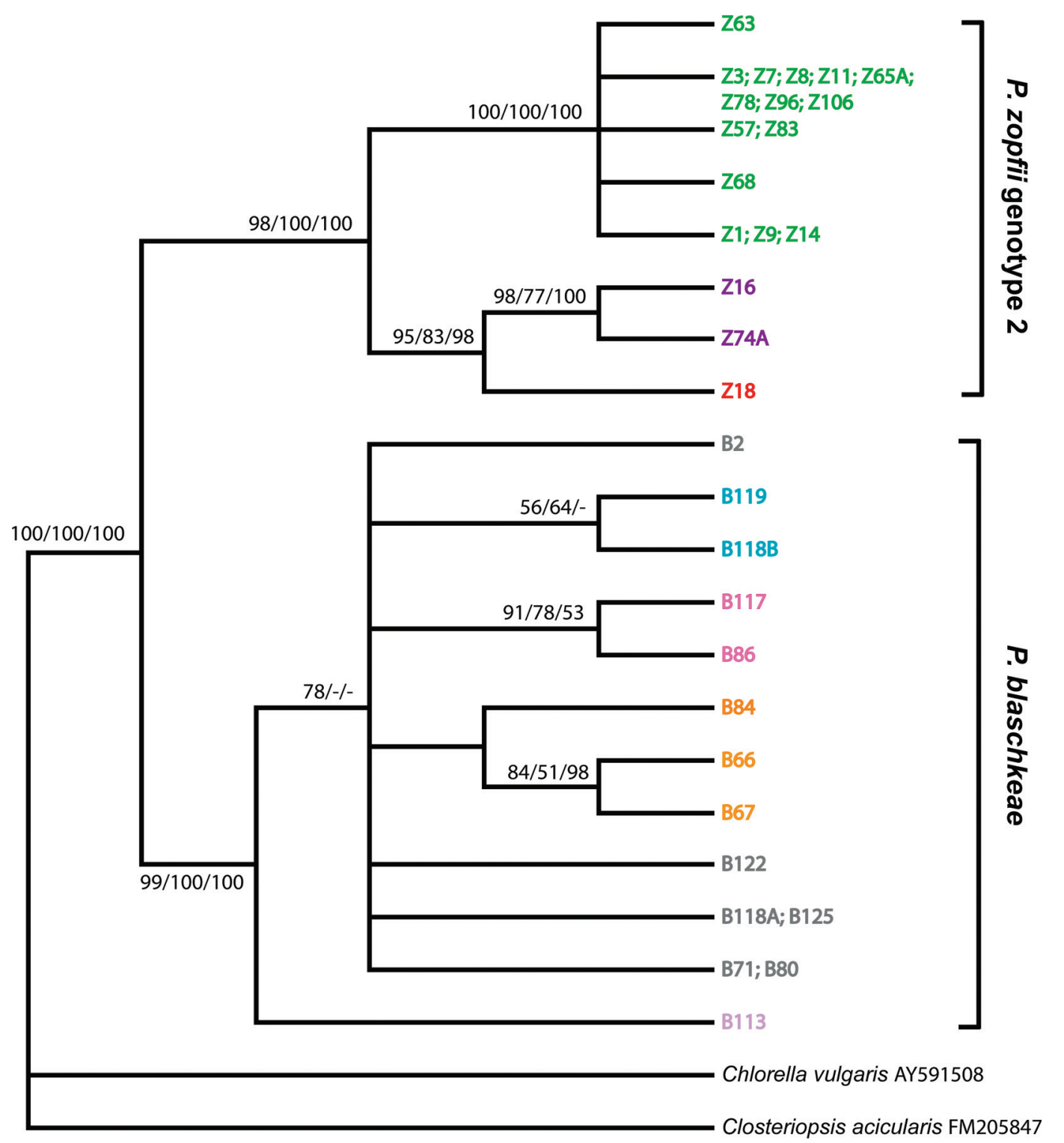

Figure 2. Phylogenetic tree inferred from complete internal transcribed space (ITS) sequences. The tree topology is derived from a maximum likelihood (ML) analysis. Bootstrap values are shown at internal nodes for each 1,000 resamplings of neighbor joining/maximum parsimony/ maximum likelihood (NJ/MP/MP) if values are higher than 50\%. Different clades within Prototheca zopfii (Z) and Prototheca blaschkeae (B) are color coded (see Figure 1). Different degrees of gray were defined to discern isolates belonging to distinct clusters as shown in Figure 1 [e.g., Z16 and Z74A (dark gray) belong to the same cluster].The ITS sequences of the related trebouxiophycean green algae Chlorella vulgaris and Closteriopsis acicularis were used as outgroups, and their GenBank accession numbers are shown. Color version available online.

sample number does not exclude mere coincidence. In contrast, 15 P. zopfii genotype 2 strains belonging to the light gray (green) clade with identical or almost identical ITS sequences were identified in herds, which were equally distributed over the whole area under study (60 km north-south vs. $19 \mathrm{~km}$ east-west; Figure 1). A similar situation was found for the distribution of $P$. blaschkeae subclades. For example, the strains of the light gray (orange) clade (B84, B66, and B67) were found in more distantly located herds, whereas indi- vidual cows of other herds in close area were infected by strains belonging to different subclades. Moreover, B80, B84, and B86 belong to different clades, although they were isolated from the same herd. From 1 herd (H1, Table 1), P. zopfii genotype 2 as well as P. blaschkeae could be isolated, whereas in all other herds from which more than 1 sample was taken, just 1 species was found, although the isolated strains often belonged to different subclades. However, a reliable correlation analysis is hampered by the fact that a possible exchange of 
Table 1. List of Prototheca zopfii (Z) and Prototheca blaschkeae (B) strains isolated in Portugal from bovine mastitic milk, their origin, herd, sample, and strain identification, and GenBank accession number of the internal transcribed space sequence

\begin{tabular}{|c|c|c|c|c|c|}
\hline Herd ID & Sample ID ${ }^{1}$ & Strain ID & Accession Number & Village & City \\
\hline H1 & $\mathrm{B} 2$ & UP-PT-P2 & HG515045 & Macieira & Vila do Conde \\
\hline \multirow[t]{3}{*}{ H5 } & $\mathrm{Z} 8$ & UP-PT-P8 & HG515037 & Afife & Viana do Castelo \\
\hline & Z9 & UP-PT-P9 & HG515033 & Afife & Viana do Castelo \\
\hline & Z11 & UP-PT-P11 & HG515035 & Afife & Viana do Castelo \\
\hline H6 & $\mathrm{Z} 18$ & UP-PT-P18 & FR848898 & S. Pedro Rates & Póvoa de Varzim \\
\hline $\mathrm{H} 22$ & $\mathrm{Z} 57$ & UP-PT-P57 & HG515042 & S. M. Coronado & Trofa \\
\hline $\mathrm{H} 23$ & Z63 & UP-PT-P63 & HG515044 & Covelas & Trofa \\
\hline $\mathrm{H} 24$ & Z65A & UP-PT-P65A & HG515038 & Arcos & Vila do Conde \\
\hline $\mathrm{H} 25$ & $\mathrm{~B} 66$ & UP-PT-P66 & HG515046 & Rio Mau & Vila do Conde \\
\hline $\mathrm{H} 26$ & B67 & UP-PT-P67 & HG515047 & Outeiro Maior & Vila do Conde \\
\hline $\mathrm{H} 27$ & Z68 & UP-PT-P68 & HG515031 & Fradelos & Vila Nova de Famalicão \\
\hline & B86 & UP-PT-P86 & HG515051 & Fajozes & Vila do Conde \\
\hline H39 & $\mathrm{Z} 83$ & UP-PT-P83 & HG515040 & Fajozes & Vila do Conde \\
\hline $\mathrm{H} 43$ & Z96 & UP-PT-P96 & HG515039 & Lavra & Matosinhos \\
\hline $\mathrm{H} 50$ & Z106 & UP-PT-P106 & HG515041 & Rio Mau & Vila do Conde \\
\hline H54 & B113 & UP-PT-P113 & HG515049 & Árvore & Vila do Conde \\
\hline H56 & B117 & UP-PT-P117 & HG515050 & Fradelos & Vila Nova de Famalicão \\
\hline \multirow{2}{*}{ H57 } & B118A & UP-PT-P118A & HG515056 & Vilar do Pinheiro & Vila do Conde \\
\hline & $\mathrm{B} 118 \mathrm{~B}$ & UP-PT-P118B & HG515048 & Vilar do Pinheiro & Vila do Conde \\
\hline \multirow[t]{3}{*}{ H58 } & B119 & UP-PT-P119 & HG515058 & Junqueira & Vila do Conde \\
\hline & B122 & UP-PT-P122 & HG515053 & Junqueira & Vila do Conde \\
\hline & $\mathrm{B} 125$ & UP-PT-P125 & HG515054 & Junqueira & Vila do Conde \\
\hline
\end{tabular}

${ }^{1}$ The sample ID was used as abbreviation in Figures 1 and 2 for Prototheca zopfii (Z) and Prototheca blaschkeae (B).

the dairy cows between different herds, resulting from trading between the dairy farmers, could not be taken into account.

\section{Development of SSPCR for Culture-Independent Identification of P. zopfii and P. blaschkeae}

The species-specific differences of the ITS sequences together with their considerable length variation allowed us to design ssPCR primer pairs for P. zopfii (PzITS-F and Pz-ITS-R) and P. blaschkeae (Pb-ITS-F and Pb-ITS-R) with PCR products of about 490 and 760 bp, respectively. These primers were tested with DNA extracted either from colonies grown on agar plates, or directly from bovine mastitis milk using a slightly modified protocol published by Cremonesi et al. (2006, 2012). We should point out, that in contrast to the amplification of the complete ITS region, no DMSO had to be added for the ssPCR. Figure 3 exemplarily shows an agarose gel with PCR products obtained from milk samples and from cultured Prototheca strains that were isolated from these samples and characterized by $18 \mathrm{~S}$ rDNA and ITS sequencing. The PCR primers clearly are specific for $P$. zopfii genotype 2 (Figure 3A) and $P$. blaschkeae (Figure 3B), and the size difference of the PCR products can easily be detected on the standard agarose gel. As negative controls, DNA extracted from milk samples of healthy cows were used (lanes 15 and 16) and 1 reaction was carried out without DNA template (lane 7). Moreover, DNA extracted from cultured $P$. zopfii genotype 1 (SAG 2063) and P. wickerhamii [isolated from systemic canine protothecosis (Tsuji et al., 2006)] failed to be amplified by both primer pairs as expected (data not shown).

\section{DISCUSSION}

The worldwide increasing incidences of bovine mastitis caused by Prototheca spp., concomitant with considerable economic losses, challenge the development of culture-independent diagnostic methods for rapid and easy identification of the major infectious pathogens, $P$. zopfii genotype 2 and P. blaschkeae. Although ribosomal ITS sequences may vary considerably between species, and therefore are widely used for species-specific identification of organisms and interspecies phylogenies 


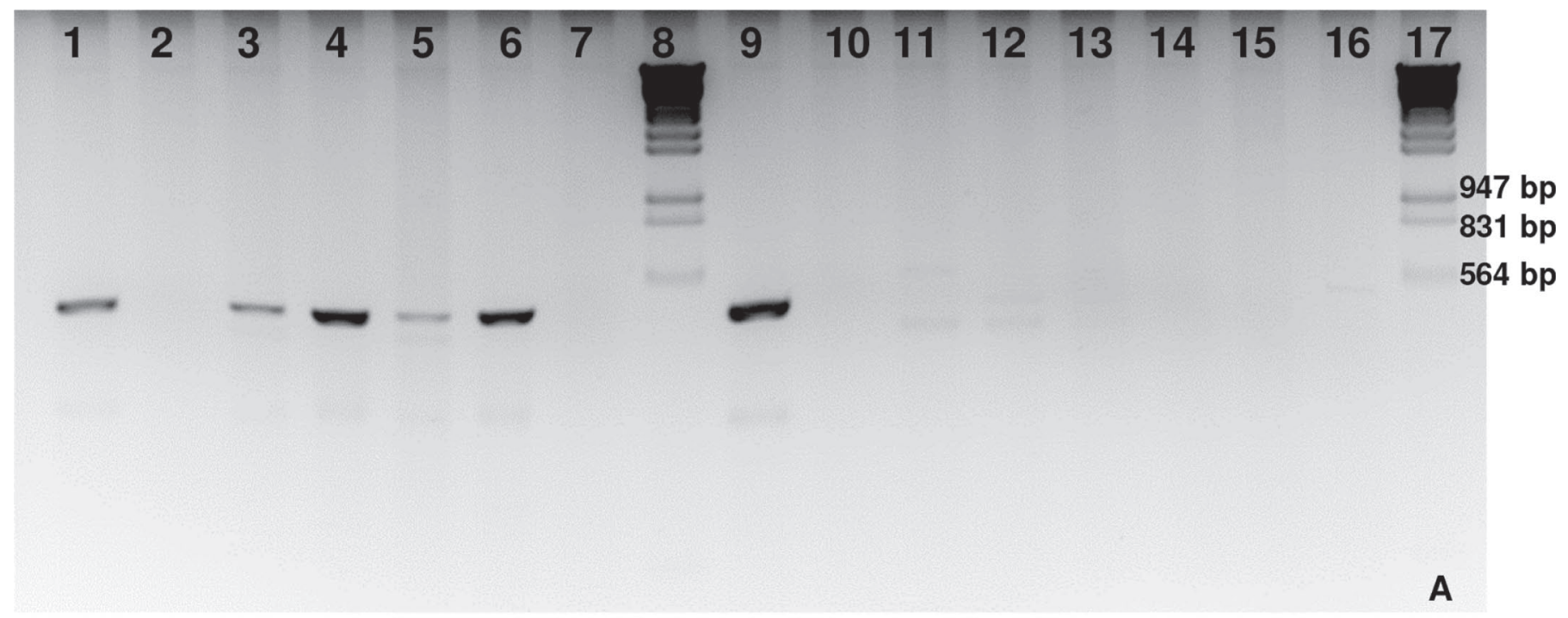

\section{$\begin{array}{lllllllllllllllll}1 & 2 & 3 & 4 & 5 & 6 & 7 & 8 & 9 & 10 & 11 & 12 & 13 & 14 & 15 & 16 & 17\end{array}$ 西
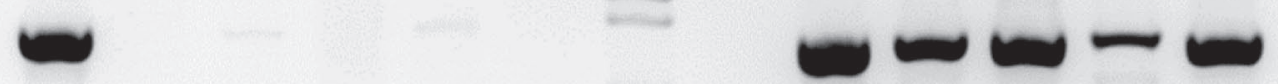

Figure 3. Species-specific PCR (ssPCR) on Prototheca isolates and on infected milk samples using species-specific internal transcribed space (ITS) PCR primers. Amplification product sizes were about 490 and 760 bp for (A) Prototheca zopfii (Z) genotype 2 and (B) Prototheca blaschkeae (B), respectively. Lanes 1 and 9, Z1 isolate; lanes 2 and 10, B2 isolate; lane 3, Z130 milk; lane 4, Z130 isolate; lane 5, Z133 milk; lane 6, Z133 isolate; lane 7, negative control without template; lanes 8 and 17, molecular weight standard; lane 11, B113 milk; lane 12, B113 isolate; lane 13, B118B milk; lane 14, B118B isolate; lanes 15 and 16, negative control with milk of healthy cows. Strain abbreviations are from Table 1.

(Coleman and Mai, 1997; Bock et al., 2010; Kamau et al., 2011), previous methods for genotyping Prototheca species from infected dairy cows were mainly based on $18 \mathrm{~S}$ ribosomal DNA (Möller et al., 2007; Marques et al., 2008). However, the conserved nature of this gene was not useful for designing ssPCR primers without additional controls or using more elaborate and expensive techniques (Möller et al., 2007; Ricchi et al., 2011; Cremonesi et al., 2012). In the current study, we modified PCR conditions, considering the exceptional GC-rich DNA base composition of Prototheca (Huss et al., 1988), by adding 5\% DMSO to the PCR reaction and could now reliably amplify the complete ITS region of 32 P. zopfii genotype 2 and P. blaschkeae isolates from infected cows in the north of Portugal. The ITS sequences not only showed considerable interspecific sequence and length variation, but also revealed intraspecific differences leading to subclades within each species in a phylogenetic analysis (Figure 2). It was tempting to see if the distribution of strains belonging to a specific subclade could be correlated to the geographical location of the herds, from which the strains were isolated (Figure 1). However, considering the relatively small sample number and missing information about a possible exchange of individual animals between herds, no obvious correlation could be detected. Nevertheless, 
our study shows that the ITS sequences even within the same Prototheca species are different enough to principally make use of such a correlation analysis for tracing infection pathways, if the critical parameters are known.

As expected, the ITS sequence alignment allowed us to design 2 ssPCR primer pairs, which can be used to discern $P$. zopfii genotype 2 and $P$. blaschkeae simply on a PCR agarose gel (Figure 3). The PCR product size difference of about $270 \mathrm{bp}$ is sufficient to obviate the need for additional controls. Moreover, this diagnostic tool can also be applied in a culture-independent assay using DNA isolated directly from milk samples of infected cows by a protocol described by Cremonesi et al. $(2006,2012)$. We verified this possibility by using DNA templates isolated from cultured strains and in parallel from the milk samples, from which the cultures were obtained (Figure 3). In each case, bands of the correct size could be detected, whereas the milk of healthy cows served as a negative control. Thus, the ssPCR primers can be used for rapid and easy identification of pathogenic Prototheca species directly from milk samples. Only recently, a paper was published by Capra et al. (2014) with the same objective. They used multiplex PCR with ssPCR primers based on chloroplast and mitochondrial sequence comparisons. In 2 PCR reactions, each 8 primers were combined to identify and distinguish P. zopfii genotype 2, P. blaschkeae, and $P$. wickerhamii by a combination of PCR products of different size on an agarose gel. Although they could clearly differentiate the Prototheca species, our protocol requires only 4 primers and uses a more convenient and widely applied molecular marker.

In summary, we present the first complete ITS sequences of 32 Prototheca zopfii genotype 2 and $P$. blaschkeae strains isolated from bovine mastitic milk of infected cows in the north of Portugal. Based on these sequences, ssPCR primers were designed for rapid and easy identification of pathogenic Prototheca directly from milk samples.

\section{ACKNOWLEDGMENTS}

This study was supported by the project "Genomics Applied to Genetic Resources" co-financed by North Portugal Regional Operational Programme 2007/2013 (ON.2 - O Novo Norte, Portugal), under the National Strategic Reference Framework (NSRF), through the European Regional Development Fund (ERDF). S. Marques was supported by Foundation for Science and Technology (Lisbon, Portugal, grant SFRH/BPD/75905/2011). The authors acknowledge Isabel Santos (Agricultural Cooperative of Vila do Conde, Portugal) and Luís Pinho (Associated Veteri- nary Services, Vila do Conde, Portugal) for providing the infected and non-infected milk samples. We also thank Júlio Carvalheira (CIBIO/UP, Vila do Conde, Portugal; ICBAS-UP, Porto, Portugal) for the technical assistance on the correlation analysis.

\section{REFERENCES}

Bock, C., T. Pröschold, and L. Krienitz. 2010. Two new Dictyosphaerium-morphotype lineages of the Chlorellaceae (Trebouxiophyceae): Heynigia gen. nov. and Hindakia gen. nov. Eur. J. Phycol. 45:267-277.

Capra, E., P. Cremonesi, C. Cortimiglia, G. Bignoli, M. Ricchi, P. Moroni, A. Pesce, M. Luini, and B. Castiglioni. 2014. Simultaneous identification by multiplex PCR of major Prototheca spp. isolated from bovine and buffalo intramammary infection and bulk tank. Lett. Appl. Microbiol. 59:642-647.

Coleman, A. W., and J. C. Mai. 1997. Ribosomal DNA ITS-1 and ITS2 sequence comparisons as a tool for predicting genetic relatedness. J. Mol. Evol. 45:168-177.

Cremonesi, P., B. Castiglioni, G. Malferrari, I. Biunno, C. Vimercati, P. Moroni, S. Morandi, and M. Luzzana. 2006. Technical note: Improved method for rapid DNA extraction of mastitis pathogens directly from milk. J. Dairy Sci. 89:163-169.

Cremonesi, P., F. Pozzi, M. Ricchi, B. Castiglioni, M. Luini, and S. Chessa. 2012. Technical note: Identification of Prototheca species from bovine milk samples by PCR-single strand conformation polymorphism. J. Dairy Sci. 95:6963-6968.

DiPersio, J. R. 2001. Prototheca and protothecosis. Clin. Microbiol. Newsl. 23:115-120.

Elwood, H. J., G. J. Olsen, and M. L. Sogin. 1985. The small-subunit ribosomal RNA gene sequences from the hypotrichous ciliates $O x y$ tricha nova and Stylonychia pustulata. Mol. Biol. Evol. 2:399-410.

Escara, J. F., and J. R. Hutton. 1980. Thermal stability and renaturation of DNA in dimethyl sulfoxide solutions: Acceleration of the renaturation rate. Biopolymers 19:1315-1327.

Gao, J., H. Q. Zhang, J. Z. He, Y. H. He, S. M. Li, R. G. Hou, Q. X. Wu, Y. Gao, and B. Han. 2012. Characterization of Prototheca zopfii associated with outbreak of bovine clinical mastitis in herd of Beijing, China. Mycopathologia 173:275-281.

Hirose, N., K. Nishimura, M. Inoue-Sakamoto, and M. Masuda. 2013 Ribosomal internal transcribed spacer of Prototheca wickerhamii has characteristic structure useful for identification and genotyping. PLoS ONE 8:e81223.

Huss, V. A. R., K. H. Wein, and E. Kessler. 1988. Deoxyribonucleic acid reassociation in the taxonomy of the genus Chlorella. Arch. Microbiol. 150:509-511.

Jagielski, T., H. Lassa, J. Ahrholdt, E. Malinowski, and U. Roesler. 2011. Genotyping of bovine Prototheca mastitis isolates from Poland. Vet. Microbiol. 149:283-287.

Jagielski, T., H. Lassa, J. Ahrholdt, U. Roesler, and E. Malinowski. 2010. Molecular characterization of Polish Prototheca zopfii mastitis isolates and first isolation of Prototheca blaschkeae in Poland. Pol. J. Vet. Sci. 13:725-729.

Jánosi, S., F. Ratz, G. Szigeti, M. Kulcsar, J. Kerenyi, T. Lauko, F. Katona, and G. Huszenicza. 2001. Review of the microbiological, pathological, and clinical aspects of bovine mastitis caused by the alga Prototheca zopfii. Vet. Q. 23:58-61.

Kamau, J., B. Salim, N. Yokoyama, P. Kinyanjui, and C. Sugimoto. 2011. Rapid discrimination and quantification of Theileria orientalis types using ribosomal DNA internal transcribed spacers. Infect. Genet. Evol. 11:407-414.

Krienitz, L., C. Bock, P. K. Dadheech, and T. Pröschold. 2011. Taxonomic reassessment of the genus Mychonastes (Chlorophyceae, Chlorophyta) including the description of eight new species. Phycologia 50:89-106.

Malinowski, E., H. Lassa, and A. Klossowska. 2002. Isolation of Prototheca zopfii from inflamed secretion of udders. Bull. Vet. Inst. Pulawy 46:295-299. 
Marques, S., E. Silva, J. Carvalheira, and G. Thompson. 2010. Phenotypic characterization of mastitic Prototheca spp. isolates. Res. Vet. Sci. 89:5-9.

Marques, S., E. Silva, C. Kraft, J. Carvalheira, A. Videira, V. Huss, and G. Thompson. 2008. Bovine mastitis associated with Prototheca blaschkeae. J. Clin. Microbiol. 46:1941-1945.

Möller, A., U. Truyen, and U. Roesler. 2007. Prototheca zopfii genotype 2: The causative agent of bovine protothecal mastitis? Vet. Microbiol. 120:370-374.

Olsen, G. J., R. Overbeek, N. Larsen, T. L. Marsh, M. J. McCaughey, M. A. Maciukenas, W.-M. Kuan, T. J. Macke, Y. Xing, and C. R. Woese. 1992. The ribosomal database project. Nucleic Acids Res. 20:2199-2200.

Onozaki, M., K. Makimura, K. Satoh, and A. Hasegawa. 2013. Detection and identification of genotypes of Prototheca zopfii in clinical samples by quantitative PCR analysis. Jpn. J. Infect. Dis. 66:383-390.

Ricchi, M., G. Cammi, C. A. Garbarino, P. Buzzini, G. L. Belletti, and N. Arrigoni. 2011. A rapid real-time PCR/DNA resolution melting method to identify Prototheca species. J. Appl. Microbiol. 110:27-34.

Ricchi, M., C. De Cicco, P. Buzzini, G. Cammi, N. Arrigoni, M. Cammi, and C. Garbarino. 2013. First outbreak of bovine mastitis caused by Prototheca blaschkeae. Vet. Microbiol. 162:997-999.

Roesler, U., and A. Hensel. 2003. Longitudinal analysis of Prototheca zopfii-specific immune responses: Correlation with disease progression and carriage in dairy cows. J. Clin. Microbiol. 41:1181-1186.

Roesler, U., A. Moller, A. Hensel, D. Baumann, and U. Truyen. 2006. Diversity within the current algal species Prototheca zopfii: A proposal for two Prototheca zopfii genotypes and description of a novel species, Prototheca blaschkeae sp. nov. Int. J. Syst. Evol. Microbiol. 56:1419-1425.

Satoh, K., K. Ooe, H. Nagayama, and K. Makimura. 2010. Prototheca cutis sp. nov., a newly discovered pathogen of protothecosis iso- lated from inflamed human skin. Int. J. Syst. Evol. Microbiol. 60:1236-1240.

Smith, K. L., J. E. Hillerton, and R. J. Harmon. 2001. Guidelines on normal and abnormal raw milk based on somatic cell counts and signs of clinical mastitis. Pages $1-3$ in National Mastitis Council Guidelines. National Mastitis Council, Madison, WI.

Sobukawa, H., S. Yamaguchi, R. Kano, T. Ito, K. Suzuki, M. Onozaki, A. Hasegawa, and H. Kamata. 2012. Short communication: Molecular typing of Prototheca zopfii from bovine mastitis in Japan. J. Dairy Sci. 95:4442-4446.

Stamatakis, A., P. Hoover, and J. Rougemont. 2008. A rapid bootstrap algorithm for the RAxML web servers. Syst. Biol. 57:758-771.

Swofford, D. L. 2002. PAUP*. Phylogenetic analysis using Parsimony (*and other methods). Version 4.0b 10. Sinauer Associates, Sunderland, MA.

Thompson, G., E. Silva, S. Marques, A. Muller, and J. Carvalheira 2009. Algaemia in a dairy cow by Prototheca blaschkeae. Med. Mycol. 47:527-531.

Tsuji, H., R. Kano, A. Hirai, M. Murakami, T. Yanai, Y. Namihira, J. Chiba, and A. Hasegawa. 2006. An isolate of Protothece wickerhamii from systemic canine protothecosis. Vet. Microbiol. 118:305-311.

Ueno, R., N. Hanagata, N. Urano, and M. Suzuki. 2005. Molecular phylogeny and phenotypic variation in the heterotrophic green algal genus Prototheca (Trebouxiophyceae, Chlorophyta). J. Phycol. 41:1268-1280.

Ueno, R., N. Urano, and M. Suzuki. 2003. Phylogeny of the nonphotosynthetic green micro-algal genus Prototheca (Trebouxiophyceae, Chlorophyta) and related taxa inferred from SSU and LSU ribosomal DNA partial sequence data. FEMS Microbiol. Lett. $223: 275-280$.

Van der Auwera, G., S. Chapelle, and R. De Wachter. 1994. Structure of the large ribosomal subunit RNA of Phytophthora megasperma, and phylogeny of the oomycetes. FEBS Lett. 338:133-136. 